\title{
Die Finanzpolitik des Bundes in den kommenden Jahren
}

von Steffen Kampeter

\begin{abstract}
Owing to the new institutional framework, Germany's public finances will emerge strengthened from the crisis. Stronger rules in conjunction with improved incentives for future policies are key in this regard. The interplay of tax revenue estimates and the debt rule made it possible to use a top-down approach of budget drafting, as the scope for expenditures is defined ex ante. The growth-supportive consolidation of the recent years in Germany pays off. The German Federal Government utilizes the newly gained financial leeway for increased spending on investment, education, research, and transport infrastructure.
\end{abstract}

Dank des neuen institutionellen Rahmenwerks gehen die öffentlichen Finanzen in Deutschland langfristig gestärkt aus der Krise hervor. Die erhöhte Regelverbindlichkeit und die Verbesserung der Eigenanreize zu einer zukunftsgerichteten Politik machen sich zunehmend bemerkbar. Das Zusammenspiel aus Steuerschätzung und Schuldenregel schafft die Möglichkeit, den Haushalt in einem echten Top-down-Verfahren aufzustellen, da der Spielraum für die Ausgabenseite ex ante definiert wird. Die wachstumsfreundliche Konsolidierung der vergangenen Jahre in Deutschland zahlt sich aus. Die Bundesregierung nutzt neu gewonnene finanzpolitische Spielräume für Investitionen in Bildung, Forschung und Verkehrsinfrastruktur.

\section{Einleitung}

Die deutsche Wirtschaft hat sich in den vergangenen Jahren in einem schwierigen internationalen Umfeld als sehr widerstandsfähig erwiesen. Sie hat den Einbruch in Folge der weltweiten Finanz- und Wirtschaftskrise 2008/2009 schneller und besser überwunden als nahezu alle vergleichbaren Industrieländer. Auch während der darauf folgenden Krise der europäischen Staatsfinanzen blieb sie auf Wachstumskurs. Die Arbeitslosigkeit liegt nun auf dem niedrigsten Stand seit 20 Jahren und die Erwerbstätigkeit bewegt sich auf historisch hohem Niveau. Die finanz- und wirtschaftspolitischen Weichenstellungen hierfür wurden in den letzten zehn Jahren gelegt. Sie haben sich positiv auf Wachstum und Beschäftigung in Deutschland ausgewirkt. Die Finanzpolitik hat mit der wachstumsfreundlichen Konsolidierung des Bundeshaushalts den Trend steigender Ver- 
schuldung durchbrochen. Die Wirtschaftspolitik hat mit strukturellen Reformen die Wettbewerbsfähigkeit der deutschen Wirtschaft weiter gestärkt. In Europa ist Deutschland heute Stabilitätsanker und Wachstumsmotor.

$\mathrm{Zu}$ dieser günstigen wirtschaftlichen Entwicklung hat die Politik der Bundesregierung das entscheidende Fundament geliefert. So hat sie die Bürgerinnen und Bürger spürbar entlastet, um Wachstumskräfte freizusetzen. Sie hat den Wettbewerb gestärkt, die Flexibilität des Arbeitsmarktes erhöht und die Rahmenbedingungen für Innovationen und Investitionen substanziell verbessert. Sie hat zudem Maßnahmen ergriffen, um die langfristigen Herausforderungen des demografischen Wandels und der Energiewende zu bewältigen.

Die wichtigsten dieser finanz- und wirtschaftspolitischen Strukturreformen sollen im Folgenden noch einmal kurz dargestellt werden, da die Finanzpolitik der 18. Legislaturperiode nur vor dem Hintergrund ihres institutionellen Rahmens zu verstehen ist.

Das zentrale Anliegen der Finanzpolitik der Bundesregierung bleibt dabei, die ökonomische Wirkungskette zu nutzen. Wachstumsfreundliche Konsolidierung und Strukturreformen insbesondere auf den Arbeits- und Produktmärkten schaffen Vertrauen bei Anlegern, Unternehmen und Bürgern in die Stabilität der Währung und in die finanzielle Handlungsfähigkeit des Staates. Dies führt zu einem Gewinn an Wachstumskraft sowie Widerstands- und Wettbewerbsfähigkeit der deutschen und damit - angesichts der engen Verflechtungen unserer Volkswirtschaften - auch der europäischen Wirtschaft.

Dieses Wachstum wiederum garantiert tragfähige öffentliche Finanzen in der Zukunft, die es uns ermöglichen, die notwendigen Zukunftsinvestitionen vorzunehmen und den demografischen Herausforderungen zu begegnen. Auch hiermit wurde bereits begonnen. Die Bundesregierung nutzt neu gewonnene finanzielle Spielräume in der 18. Legislaturperiode für prioritäre Maßnahmen insbesondere in den Bereichen Infrastruktur, Forschung und Bildung. Zudem hat der Bund die Kommunen in den vergangenen Jahren finanziell umfangreich entlastet und damit auch die Investitionskraft des bedeutendsten öffentlichen Investors in Deutschland substanziell verbessert.

\section{Stabile wirtschaftspolitische Basis}

Dass Deutschland die Rolle des „kranken Mann in Europa“ abgestreift hat, ist ein Verdienst der tiefgreifenden Strukturreformen, denen sich die Bundesrepub- 
lik nach der Jahrtausendwende gestellt hat. ${ }^{1}$ Diese Reformen haben langfristige Wachstumsimpulse gesetzt, von denen Deutschland heute profitiert.

Für die Finanzpolitik des Bundes sind zwei Elemente der Reformpolitik von besonderer Bedeutung. Die Hartz-Reformen der Arbeitsmarkt- und Sozialpolitik haben - flankiert durch eine verantwortungsbewusste Lohnpolitik - zu einem nachhaltigen Beschäftigungsaufbau geführt, der sich positiv auf die Einnahmen und Ausgaben des Staates auswirkt. So lässt sich laut einer aktuellen Studie des Internationalen Währungsfonds eine Reduzierung der Arbeitslosenquote um ca. 1,4 Prozentpunkte auf die Hartz-IV-Reform zurückführen. ${ }^{2}$

Die Einnahmesituation aller Sozialversicherungssysteme sowie von Bund, Ländern und Gemeinden wurde gestärkt und insbesondere die Ausgaben der Arbeitslosenversicherung wurden reduziert. ${ }^{3}$ Zudem hat die Rentenreform - Stichwort Rente mit 67 - die gesetzliche Rentenversicherung zukunftsfest gestaltet und die sozialen Sicherungssysteme auf die anstehenden demografischen Entwicklungen vorbereitet.

Diese Reformpolitik hat die Bundesregierung zu Beginn der 17. Legislaturperiode mit Hilfe des Wachstumsbeschleunigungsgesetzes fortgeführt. Insbesondere die mittelständischen Unternehmen wurden - allein in 2010 um 8,5 Milliarden Euro - entlastet. Hierbei ist vor allem die Beseitigung vieler „krisenverschärfender Elemente" der Unternehmensbesteuerung von Bedeutung gewesen.

Mit dem Energiekonzept hat sich die vorangegangene Bundesregierung ambitionierte energie- und klimapolitische Ziele gesetzt: Erstens soll der Ausstoß der Treibhausgasemissionen bis 2050 um mindestens 80 \% gegenüber 1990 reduziert werden. Zweitens sollen die erneuerbaren Energien zukünftig den Hauptanteil der deutschen Energieversorgung ausmachen. Und drittens soll der Energieverbrauch deutlich gesenkt und die Energieeffizienz erhöht werden.

Damit steht die Energieversorgung der Bundesrepublik Deutschland vor einem fundamentalen Umbau. Zugleich sind die deutschen Haushalte und Unternehmen auch in Zukunft auf eine sichere und bezahlbare Energieversorgung angewiesen. Nur so kann Deutschland ein wettbewerbsfähiger Industriestandort bleiben. Mit einem umfangreichen Gesetzespaket, dem so genannten Energiepaket, wurden

1 Vgl. Eichhorst, W.: Vom kranken Mann zum Vorbild Europas: Kann Deutschlands Arbeitsmarkt noch vom Ausland lernen?, in: IZA Standpunkte, 46 (2011).

2 Vgl. Krebs, T./Scheffel, M.: Macroeconomic Evaluation of Labor Market Reform in Germany, in: IMF Working Paper 13/42 (2013).

3 Für eine weitere Abschätzung der makroökonomischen Effekte der Hartz-Reformen siehe Bräuninger, M./Michaelis, J./Sode, M.: 10 Jahre Hartz-Reformen, in: HWWI Policy Paper, 73 (2013). 
im Sommer 2011 die Grundlagen für einen schrittweisen Ausstieg aus der Kernenergie bis 2022 sowie für die Beschleunigung des Netzausbaus und den weiteren Ausbau der erneuerbaren Energien gelegt. Dies sichert die deutsche Wirtschaft auch langfristig gegen geopolitische Risiken ab.

\section{Weiterentwicklung der Fiskalregeln}

Während die Reformpolitik die deutsche Wirtschaft gegen Wirtschaftskrisen festigte, zeigte sich im Zuge der Finanz- und Staatsschuldenkrise nach 2007, dass die bisherigen fiskalischen Regeln nicht ausreichten, um langfristig stabile und tragfähige öffentliche Haushalte zu garantieren. Die Beiträge etwa der Ökonomen Reinhardt und Rogoff haben herausgearbeitet, dass langfristig hohe Staatsschuldenquoten in hohem Maße schädlich für die ökonomische Entwicklung einer Volkswirtschaft sind. ${ }^{4}$ Eine Absicherung gegen die inhärenten Boomund Krisenzyklen des Wirtschaftsgeschehens ist demnach nicht ausreichend, um langfristige Prosperität in Deutschland und Europa zu garantieren.

Wenn man sich der Geschichte der Staatsfinanzen zuwendet, so stellt man fest, dass sie zugleich auch die Geschichte der ewigen Versuchung ist. Schon der schottische Philosoph David Hume bemerkte vor gut zweihundertfünfzig Jahren mit großem Weitblick:

„Für einen Minister ist es sehr verführerisch, ein derartiges Mittel [die Staatsschuld, Anm. d. Verf.] zu benutzen, das ihn in den Stand setzt, während seiner Verwaltung den großen Mann zu spielen, ohne das Volk mit Steuern zu überladen, oder eine sofortige Unzufriedenheit gegen sich zu erregen. Die Praxis des Schuldenmachens wird daher fast unfehlbar von jeder Regierung missbraucht werden. “5

$\mathrm{Zu}$ welchen Ergebnissen dies führen kann, zeigte sich leidvoll in den vergangenen Jahrzehnten. Regeln zur Begrenzung der Staatsschuld erwiesen sich als löchrig. Ausnahmeregelungen, wie im früheren Artikel 115 des Grundgesetzes (GG) für den Fall einer „Störung des gesamtwirtschaftlichen Gleichgewichts“, waren unbestimmt und wurden überdehnt. In Kombination mit einer mangelnden Regeltreue, auch durch Deutschland, häuften die europäischen Staaten hohe Schuldenstände an, trotz des Maastrichter Vertrages. Verschärfend wirkte, dass bestehende Frühwarnsysteme lückenhaft waren und ihre Signale nicht genügend ernst genommen wurden.

4 Vgl. Reinhart, C.M./Rogoff, K.S.(2010): Growth in a Time of Debt, in: American Economic Review, 100,2 (2010), 573-78.

5 Vgl. Hume, D.: Nationalökonomische Abhandlungen, 1751, zitiert nach Blankart, C.: „Öffentliche Finanzen in der Demokratie“, München, 2011. 
Aus ökonomischer Sicht sprechen gewichtige Gründe dafür, die Höhe der Staatsverschuldung auf ein tragfähiges $\mathrm{Ma} ß$ zu begrenzen. Solide öffentliche Finanzen garantieren die finanzielle Handlungsfähigkeit des Staates; dagegen würde eine stetig steigende Zinsbelastung diesen Handlungsspielraum immer weiter einschränken. Darüber hinaus spielen Vertrauenseffekte eine wichtige Rolle: Tragfähige Finanzen stärken die Erwartungen der Finanzmärkte, der Unternehmen und der Bürger in stabile Preise, niedrige Zinsen und kalkulierbare Steuerlasten; dies erhöht die Investitions- und Anschaffungsneigung. Nicht zuletzt geht es auch darum, positive Rückkopplungseffekte zwischen dem Abbau der Staatsverschuldung und dem Wirtschaftswachstum nutzbar zu machen. Denn einerseits sind solide Finanzen gut für das Wachstum, andererseits erleichtert nachhaltiges Wachstum die Rückführung der Verschuldung.

Von zentraler Bedeutung ist daher die Frage, welche institutionellen Rahmenbedingungen dazu geeignet sind, der Problematik einer stetig ansteigenden Staatsverschuldung entgegenzuwirken. In diesem Zusammenhang sind Fiskalregeln ein wichtiges Instrument, da sie einen Beitrag zur Reduzierung der Verschuldungsanreize liefern können. Fiskalregeln sollen staatliche Verschuldungsmöglichkeiten begrenzen und Verstöße mit Sanktionen belegen. Ziel der Regeln ist also ein „Selbstschutz“, der verhindert, dass heute im Übermaß Entscheidungen getroffen werden, die langfristig schädlich für die öffentlichen Finanzen sein werden.

Der gesamtstaatliche Schuldenstand Deutschlands lag noch bis zum Jahr 2012 bei Werten von über $80 \%$ in Relation zum Bruttoinlandsprodukt (BIP). ${ }^{6}$ Ein Blick zurück macht deutlich, dass dieser - zumal angesichts der demografischen Entwicklung - auf Dauer inakzeptabel hohe Wert nicht allein durch die Auswirkungen der Wirtschafts- und Finanzkrise in den Jahren 2009 und 2010 oder durch die Finanzierung der deutschen Einheit nach 1990 erklärt werden kann. Betrachtet man die Entwicklung der gesamtstaatlichen Verschuldung seit dem Jahr 1950, so zeigt sich, dass die Staatsverschuldung in den ersten Jahrzehnten der Bundesrepublik gleichbleibend in einer Größenordnung von rund $20 \%$ in Relation zum BIP lag. Die Schuldenstandsquote schwankte zwar im Zeitablauf, sie wies jedoch in den Jahren 1950 bis 1970 keinen steigenden Trend auf. Für den Bund allein betrachtet lag der Schuldenstand noch bis 1973 unter 10\% in Relation zum BIP.

6 Der starke Anstieg der Schuldenstandsquote von 2008 bis 2012 ging insbesondere darauf zurück, dass die neu errichteten Abwicklungsanstalten der Hypo Real Estate und der WestLB dem Sektor Staat zugeordnet wurden und somit in den Schuldenstand einflossen. 
Erst seit Anfang der 1970er Jahre ist die Staatsverschuldung kontinuierlich gewachsen. Dabei kommt der im Jahr 1969 im Rahmen der Haushalts- und Finanzreform erfolgten Änderung des Artikel 115 GG im Einklang mit der damals aufkommenden Neigung zum Versuch konjunktureller Feinsteuerung besondere Bedeutung zu: Aufgrund dieser Neuregelung bestand fortan die Möglichkeit, (Brutto)-Investitionen über Verschuldung zu finanzieren und diskretionäre finanzpolitische Maßnahmen zu ergreifen.

Entgegen ökonomischer Grundsätze beschränkte sich die aktive Konjunkturpolitik der folgenden Jahre allerdings auf konjunkturell ungünstige Zeiten (Ausweitung der Nettokreditaufnahme), ohne entsprechende Rückführung der Schuldenstandsquote in Aufschwung- und Boomphasen. Darüber hinaus kam die damalige Fiskalregel ausschließlich bei der Haushaltsaufstellung zur Anwendung - im tatsächlichen Vollzug des Haushalts fand keine Überprüfung der Regel statt. Somit konnte eine Verletzung der Regel ex post nicht mit Sanktionen einhergehen. Zudem war die vorgesehene Ausnahmeregelung nicht restriktiv genug ausgestaltet, sondern durch Erklärung einer ,Störung des gesamtwirtschaftlichen Gleichgewichts“ - wie rückblickend festzustellen ist - allzu leicht nutzbar. Somit kam es zu einem ansteigenden Trend der Schuldenstandsquote, der sich aus den 1970er Jahren bis in die heutige Zeit fortgesetzt hat. Allein der Schuldenstand des Bundes stieg bis zum Jahr 2009 auf über 40 \% des BIP. Die Staatsverschuldung hatte somit bereits vor einigen Jahren eine Größenordnung erreicht, die einen dringenden Handlungsbedarf verdeutlichte, um zukünftig eine tragfähige Entwicklung der Schuldenstandsquote sicherzustellen.

\section{Die grundgesetzliche Schuldenregel ${ }^{7}$}

Die seither angehäuften Staatsschulden machten in Deutschland und Europa eine entschlossene Stärkung der Widerstandskräfte gegen die Versuchung der Verschuldung unausweichlich. Deshalb hat die große Koalition unter Führung von Bundeskanzlerin Dr. Angela Merkel im Jahr 2009 - im Zuge der Föderalismuskommission II - die Initiative für die Verankerung einer neuen Schuldenregel im Grundgesetz ergriffen. Diese gibt einen verbindlichen Rahmen für die schrittweise Rückführung der strukturellen Haushaltsdefizite vor. Weithin positiv beurteilt, diente die deutsche Schuldenregel auch als Vorbild für vergleichbare Reformen in anderen Ländern des Euroraums. So hält beispielsweise der deutsche Sachver-

7 Siehe hierzu auch Kampeter, S. (2013): Solide Staatsfinanzen in Deutschland und Europa, in: Pies, I.(Hg.): Das weite Feld der Ökonomik - von der Wirtschaftsforschung und Wirtschaftspolitik bis zur Politischen Ökonomik und Wirtschaftsethik, Stuttgart, 2013, 219-229. 
ständigenrat die neue Regel für eine „finanzpolitische Errungenschaft“, die „einen wichtigen und richtigen Beitrag zur wirksamen Begrenzung der staatlichen Verschuldung" leistet. ${ }^{8}$

Die Schuldenregel führt dazu, dass die Konsolidierung der Staatsfinanzen bei den politischen Entscheidungsträgern zusätzliches Gewicht erhält. An dem im Juni 2014 vom Deutschen Bundestag beschlossenen Bundeshaushalt 2014, der ohne strukturelle Neuverschuldung auskommt, und insbesondere auch an dem am 2. Juli 2014 vom Kabinett beschlossenen Regierungsentwurf zum Bundeshaushalt 2015 und zum Finanzplan für den Zeitraum bis 2018, in denen die Haushalte in allen Jahren ohne Neuverschuldung ausgeglichen werden, lässt sich ablesen, dass die grundgesetzliche Schuldenregel wirkt.

Für die Wirksamkeit von Fiskalregeln ist eine Vielzahl von Parametern entscheidend. Von der konkreten Ausgestaltung solcher Regeln hängt entscheidend ab, ob die beabsichtigte Wirkung erzielt werden kann. Exemplarisch seien einige notwendige Bedingungen genannt, die eine wirksame Fiskalregel erfüllen muss:

- Glaubwürdigkeit der Regel: Dazu gehört insbesondere eine stabile rechtliche Grundlage. Erstrebenswert ist eine Regel mit Verfassungsrang, da eine einfache Gesetzgebung keine zwingende Verpflichtung für künftige Regierungen und Parlamente bedeuten muss.

- Verpflichtung zur Erzielung von Überschüssen in ,guten Zeiten“: Eine solide Fiskalpolitik braucht genügend Spielraum, um in konjunkturell ungünstigen Zeiten temporäre Defizite hinzunehmen. Allerdings müssen diesen Defiziten in konjunkturell günstigen Zeiten entsprechende Überschüsse gegenüberstehen, die eine Rückführung der Schuldenstandsquote gewährleisten.

- Vollzugskontrollen: Eine wirksame Regel muss sowohl ex ante (bei Aufstellung des Haushalts) als auch ex post (nach Abschluss eines Haushaltsjahres) Anwendung finden. Nur so ist es möglich, Regelverstöße, die sich erst im Haushaltsvollzug ergeben, zu erfassen.

- Korrektur von Regelverstößen: Abweichungen von der Schuldenregel müssen korrigiert werden, indem die übermäßige Neuverschuldung gezielt getilgt wird.

- Ausreichende Flexibilität in Krisenfällen: Ausnahmeklauseln für Notsituationen sind vorzusehen.

8 Vgl. Sachverständigenrat zur Begutachtung der gesamtwirtschaftlichen Entwicklung: Jahresgutachten 2010/11, Textziffer 360, 2010. 
- Starke öffentliche Verankerung: Zusätzlich zu den gesetzlichen Vorgaben spielen auch die politischen Kosten bei Nichteinhaltung einer Fiskalregel eine wichtige Rolle. Wenn die Öffentlichkeit hinreichend informiert ist über die zentrale Bedeutung solider Staatsfinanzen, kann ein Verstoß gegen die Schuldenregel durch den Wähler sanktioniert werden.

Die deutsche Schuldenregel ist so konzipiert worden, dass sie diese Kriterien für eine ,gute“ Fiskalregel erfüllt und somit dazu beiträgt, finanzpolitische Fehler der Vergangenheit zukünftig deutlich zu erschweren. Wesentliche Elemente der Schuldenregel sind: ${ }^{9}$

- Die Ausgestaltung der Schuldenregel zielt auf strukturell nahezu ausgeglichene Haushalte. Sie gibt vor, in welcher Höhe eine Nettokreditaufnahme durch den Bund maximal zulässig ist. Dabei ist in konjunkturell normalen Zeiten eine maximale Verschuldung in Höhe einer Strukturkomponente von maximal 0,35 \% des BIP (derzeit rund 10 Milliarden Euro) zulässig.

- Die Schuldenregel wirkt über den Konjunkturverlauf symmetrisch. Zusätzlich zur Strukturkomponente wird eine Konjunkturkomponente berechnet, die eine symmetrische Berücksichtigung der konjunkturellen Lage gewährleistet. So werden konjunkturell günstige Zeiten dafür genutzt, die Defizite nachhaltig zurückzuführen und auf ein dauerhaft tragfähiges Maß zu begrenzen. Damit tragen die Vorgaben der Schuldenregel entscheidend zu langfristig stabilen Rahmenbedingungen bei. ${ }^{10}$ Darüber hinaus erfolgt eine Bereinigung um den Saldo der finanziellen Transaktionen.

- Es erfolgt eine ex post Überprüfung der tatsächlichen Neuverschuldung eines Jahres. Um eine Überprüfung nach Abschluss des Haushaltsjahres sicherzustellen, wurde ein Kontrollkonto eingerichtet, auf dem Abweichungen von den Vorgaben der Schuldenregel erfasst werden, die sich im Haushaltsvollzug ergeben haben.

- Für außergewöhnliche Notsituationen ist eine Ausnahmeregelung vorgesehen. Diese Konstruktion ist notwendig, um beispielsweise im Falle von Naturkatastrophen handlungsfähig zu bleiben, ohne gegen die grundgesetzliche Schuldenregel zu verstoßen. Gleichzeitig muss ein Tilgungsplan verabschiedet werden, der die Rückführung der oberhalb der Regelgrenze liegenden Nettokreditaufnahme innerhalb eines angemessenen Zeitraums vorsieht.

9 Weitergehende ausführliche Erklärungen zur Schuldenregel bietet Bundesministerium der Finanzen: Kompendium zur Verschuldungsregel des Bundes gemäß Artikel 115 Grundgesetz, (2012), abrufbar unter: http://www.bundesfinanzministerium.de.

10 Detaillierte Informationen zum Konjunkturbereinigungsverfahren der Schuldenregel finden sich unter Bundesministerium der Finanzen (2011): Die Ermittlung der Konjunkturkomponente des Bundes im Rahmen der neuen Schuldenregel, in: BMF-Monatsbericht Februar (2011), abrufbar unter: http://www.bundesfinanzministerium.de. 
Die Schuldenregel soll dazu führen, dass die Konsolidierung der Staatsfinanzen sowohl in der Öffentlichkeit als auch seitens der politischen Entscheidungsträger als Priorität angesehen wird. Dies ist zugleich ein Beitrag zur Sicherung des Vertrauens in tragfähige Staatsfinanzen. Auch angesichts der entstandenen Unsicherheiten hinsichtlich der Lösung der Verschuldungsprobleme im Euroraum besteht die derzeitige Hauptaufgabe der Wirtschafts- und Finanzpolitik darin, das Vertrauen von Konsumenten, Unternehmen und Märkten in langfristig tragfähige Staatsfinanzen zu sichern. Entscheidend ist dabei die konkrete Umsetzung der Schuldenregel im Budgetprozess.

Im Übergangszeitraum bis Ende 2015 und natürlich auch danach gilt es, eine stetige und konjunkturgerechte Haushaltspolitik sicherzustellen. Das beinhaltet vor allem, dass die vorgegebenen Defizitgrenzen des Abbaupfads nicht ausgereizt werden - vielmehr muss ein ausreichender Sicherheitsabstand zur maximal zulässigen Neuverschuldung eingehalten werden. Ein solcher wurde von der Bundesregierung - sowohl unter der christlich-liberalen Koalition als auch der zweiten großen Koalition unter Bundeskanzlerin Dr. Angela Merkel - in den letzten Jahren stets eingehalten. Entsprechend der Eckwerte für die Finanzplanung 2015 bis 2018 ist dies auch weiterhin vorgesehen. ${ }^{11}$

Abbildung 1: Grundgesetzliche Schuldenregel. Strukturelle Nettokreditaufnahme in $\%$ des BIP

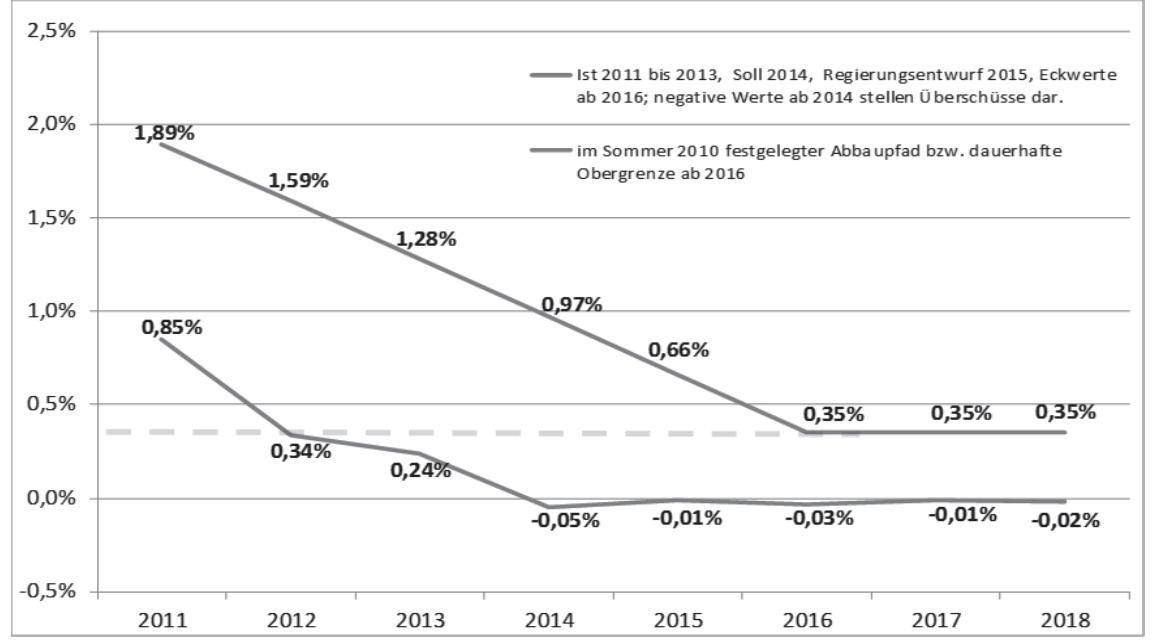

Quelle: BMF, Juli 2014.

11 Vgl. hierzu $A b b .1$. 
Die noch ausstehenden Ausgaben des Fonds „Aufbauhilfe“ zur Behebung von Schäden des Hochwassers im Mai und Juni 2013 werden hier nicht berücksichtigt. Es ist derzeit noch nicht absehbar, in welchem Zeitraum und mit welchen Jahresfälligkeiten die übrigen Mittel des Fonds verausgabt werden.

Doch Konsolidierung ist mehr als Buchhaltung. Es geht nicht um mechanische Defizitreduktion, sondern um klare Prioritätensetzung. Zukunftsweisende Konsolidierung beruht auf Strukturreformen und ist immer auch Ausdruck einer wachstumsorientierten Wirtschafts- und Finanzpolitik. Konsequente Konsolidierung ist die Grundvoraussetzung für dauerhaftes Wirtschaftswachstum.

Die Ökonomen Alesina und Ardagna haben die makroökonomischen Effekte fiskalischer Konsolidierungspolitik untersucht. ${ }^{12}$ Ihre Studie kommt zu dem Ergebnis, dass vorwiegend ausgabenbasierte Konsolidierungsstrategien tendenziell

- $\quad$ zu einer nachhaltigeren Verringerung der Schuldenstandsquote führen;

- $\quad$ mit weniger ausgeprägten Rezessionen verbunden sind;

- positive Wachstumseffekte (sogenannte nicht-keynesianische Effekte) mit sich bringen können, vor allem wenn sie mit wachstumsorientierten Strukturreformen im Bereich der Arbeits- und Produktmärkte flankiert werden.

Die empirischen Ergebnisse von Alesina und Ardagna unterstreichen die Richtigkeit des europäischen Ansatzes einer wachstumsfreundlichen Konsolidierung. ${ }^{13}$ Insbesondere in den ehemaligen Programmländern Irland und Portugal sowie dem derzeitigen Programmland Griechenland wird eine Doppelstrategie aus Konsolidierung und strukturellen Reformen verfolgt, um das Marktvertrauen wiederherzustellen und das langfristige Wachstumspotenzial zu stärken. Der Ansatz verbindet - möglichst ausgabenbasierte - fiskalische Anpassungen mit den Regelungen des Stabilitäts- und Wachstumspakts mit wirtschaftspolitischen Strukturreformen zur Verbesserung der Wettbewerbsfähigkeit. Auch Berechnungen der Europäischen Zentralbank, veröffentlicht im EZB-Monatsbericht Dezember 2012, zeigen, dass die langfristigen Multiplikatoren von Konsolidierungmaßnahmen positiv sind. ${ }^{14}$

12 Alesina, A./Ardagna, S.: The Design of Fiscal Adjustments, NBER Chapters, in: Tax Policy and the Economy, National Bureau of Economic Research, Inc., 27 (2013), 19-67.

13 Vgl. auch Vortrag von Alesina, A.: Reducing Budget Deficits after the Great Recession, in: Bundesministerium der Finanzen v 15.06.2011.

14 Vgl. Europäische Zentralbank: Monatsbericht Dezember 2012, Kasten 6 (2012). 
Für die Wirkung von Strukturreformen ist es wichtig, dass Arbeitsmarkt- und Produktmarktreformen gemeinsam umgesetzt werden, da sie - als komplementäre Maßnahmen - im Zusammenspiel bessere Effekte erzielen: Arbeitsmarktreformen erhöhen das Arbeitsangebot; gleichzeitig steigern Produktmarktreformen durch Anreize für zusätzliche Investitionen die Arbeitsnachfrage; zusammen führen sie zu höherer Beschäftigung. Konsequente Strukturreformen sind der richtige Weg zur nachhaltigen Stärkung des Wirtschaftswachstums. Mit der europäischen Wachstumsstrategie Europa 2020 und den länderspezifischen Empfehlungen gibt es dafür auf europäischer Ebene das Instrumentarium, welches konsequent genutzt werden muss, besonders im Hinblick darauf, dass Strukturreformen für ihre Wirkung Zeit brauchen. Zusammen genommen bilden Wirtschaftswachstum, Wettbewerbsfähigkeit und Konsolidierung der öffentlichen Haushalte einen ,virtuous circle“, der die Chancen für Schuldenabbau und Rückkehr zu soliden Staatsfinanzen entscheidend verbessert. ${ }^{15}$

Finanzpolitischer Zwilling der Konsolidierung ist eine verlässliche und stabile Steuerpolitik, die Deutschlands internationale Wettbewerbsfähigkeit sichert. Mit dem Verzicht auf Steuererhöhungen schafft die Bundesregierung Planungssicherheit und setzt weiterhin die richtigen Investitions- und Leistungsanreize. Handlungsschwerpunkte in der Steuerpolitik der laufenden Legislaturperiode sind die Vereinfachung des Steuerrechts, die Bekämpfung der - illegalen - Steuerhinterziehung und die Eindämmung der - legalen - Steuervermeidung.

Um die Möglichkeiten der Steuervermeidung zu reduzieren, wird die Bundesregierung intensiv mit ihren europäischen und außereuropäischen Partnern zusammenarbeiten. Eine entsprechende OECD-Initiative wird von Deutschland aktiv vorangetrieben und soll 2015 Ergebnisse zur Frage der fairen Besteuerung multinational tätiger Unternehmen liefern. Zusätzlich ist die Transparenz zwischen den einzelnen nationalen Steuerbehörden auszubauen. Ein automatischer Informationsaustausch zwischen den Steuerbehörden, auf den die Bundesregierung hinarbeitet, trägt erheblich zu einem fairen Steuerwettbewerb und zur Vermeidung von Steuerhinterziehung bei.

15 Zur Bedeutung von Wachstum für Beschäftigung, Lebensqualität und soziale Sicherheit vgl. Paqué, KH.: Wachstum - Die Zukunft des globalen Kapitalismus, München, 2010. 


\section{Komplemente der Schuldenregel: Eckwerte-Verfahren und Stabi- litätsrat}

Bereits bei der konzeptionellen Entwicklung der neuen verfassungsrechtlichen Schuldenregel war klar, dass diese neue Fiskalregel auch ein geändertes Haushaltsaufstellungsverfahren verlangt. Mit der neuen Schuldenregel steht frühzeitig fest, wie hoch die maximal zulässige Neuverschuldung sein darf. Die innerhalb dieser Obergrenze definierte Höhe der für die nächsten Jahre eingeplanten Neuverschuldung und der Einzelplanbudgets steht jetzt also am Anfang des Verfahrens - und nicht mehr an dessen Ende. Mittlerweile hat die Bundesregierung zum vierten Mal einen Regierungsentwurf vorgelegt, der im Rahmen eines Eckwerte (,Top-down“) -Verfahrens aufgestellt wurde. Es ist dabei stets gelungen, die im März beschlossenen Eckwerte einzuhalten. Dies zeigt, dass dieses wichtige haushaltspolitische Steuerungsinstrument mittlerweile eine hohe Bindungswirkung entfaltet und sich für eine nachhaltige und solide Finanzpolitik bewährt hat.

Neben der Schuldenregel hat Deutschland mit dem Stabilitätsrat von Bund und Ländern bereits vor einigen Jahren einen weiteren institutionellen Pfeiler zur Sicherung der Konsolidierung der öffentlichen Haushalte verfassungsrechtlich verankert. Der Stabilitätsrat überwacht regelmäßig die Haushalte von Bund und Ländern. Hierdurch sollen drohende Haushaltsnotlagen frühzeitig erkannt und durch Sanierungsmaßnahmen der betroffenen Gebietskörperschaft abgewendet werden. Durch das im Juli 2013 in Kraft getretene Gesetz zur innerstaatlichen Umsetzung des Fiskalvertrags wird die Rolle des Stabilitätsrats nun weiter gestärkt und dieser explizit damit beauftragt, die Einhaltung der nach den europäischen Vorgaben zulässigen Obergrenze eines strukturellen gesamtstaatlichen Defizits von $0,5 \%$ des BIP zu überwachen. Falls notwendig, empfiehlt der Stabilitätsrat Bund und Ländern geeignete Konsolidierungsmaßnahmen.

Bei der Wahrnehmung seiner neuen Aufgabe wird der Stabilitätsrat durch einen unabhängigen Beirat unterstützt, dem neben weiteren Sachverständigen Vertreter der Deutschen Bundesbank, des Sachverständigenrats zur Begutachtung der gesamtwirtschaftlichen Entwicklung und an der Gemeinschaftsdiagnose beteiligte Forschungsinstitute angehören. Stellungnahmen und Empfehlungen des Beirats werden - wie die des Stabilitätsrats - veröffentlicht. Dadurch werden Glaubwürdigkeit und Transparenz des fiskalpolitischen Regelwerks weiter gestärkt. 


\section{Der gestärkte Stabilitäts- und Wachstumspakt}

Durch seine nationalen Anstrengungen leistet Deutschland einen wichtigen Beitrag zur Konsolidierung in Europa und wird damit seiner Verantwortung in der Europäischen Union gerecht. Wegen der intensiven wirtschaftlichen Verflechtungen, vor allem mit den Nachbarstaaten in Europa, greifen rein nationale Maßnahmen aber zu kurz. Auch auf europäischer Ebene müssen die richtigen Konsequenzen gezogen werden. Um die Europäische Union zu einer echten Stabilitätsunion zu vertiefen, setzt die Bundesregierung auf eine Doppelstrategie aus Solidität und Solidarität.

Solidität bedeutet die dauerhafte Rückführung der überhöhten Verschuldung und Rückgewinnung der finanziellen Handlungsfähigkeit des Staates, gepaart mit strukturellen Reformen zur Wiedergewinnung der Wettbewerbsfähigkeit der jeweiligen Wirtschaft. Künftigen Krisen wird damit effektiver vorgebeugt.

Solidarität heißt, dass „Feuerwehr“-Mechanismen bereitstehen, die helfen einen möglichen Krisenfall wirksam zu bewältigen. Für einen dauerhaft stabilen Euro sind die drei Säulen „Stabile Haushalte“, „Stabile Wirtschaft“ und „Stabile Finanzmärkte" entscheidend.

Durch die Einführung des Europäischen Semesters und die Verabschiedung der sogenannten „Six Packs“ und „Two Packs“ wurde die haushaltspolitische Überwachung erweitert. So wird z.B. der präventive Arm des Stabilitäts- und Wachstumspakts mit der Forderung nach strukturell ausgeglichenen Haushalten gestärkt und mit Sanktionsmöglichkeiten belegt.

Daneben ist eine Verpflichtung zur Rückführung des Schuldenstands auf $60 \%$ in Relation zum BIP in den korrektiven Arm des Pakts aufgenommen worden. Nicht zuletzt greifen Sanktionen zukünftig schneller und Sanktionsverfahren werden automatisiert eingeleitet. Des Weiteren findet eine Angleichung der Haushaltszyklen statt. Euro-Mitgliedstaaten legen bis Mitte Oktober eine Übersicht über ihre gesamtstaatliche Haushaltsplanung für das Folgejahr vor. Dies ermöglicht eine noch stärker präventiv wirkende Haushaltsüberwachung auf europäischer Ebene. Die Europäische Kommission prüft die Zahlen und fordert die Mitgliedstaaten bei schwerwiegenden Verstößen gegen die Defizit- und Schuldenstandsregeln zur Überarbeitung auf.

Über diese Regelungen hinaus setzt der 2013 in Kraft getretene Fiskalvertrag an einer verstärkten finanz- und wirtschaftspolitischen Koordinierung an. Durch diesen Pakt wird die finanzpolitische Stabilitätsunion auf eine verlässliche Grundlage auch in den Vertragsstaaten gestellt. Der Vertrag beinhaltet die Vor- 
gabe nationale Schuldenregeln vorzugsweise mit Verfassungsrang einzuführen. Diese sollen höchstens ein strukturelles Defizit von 0,5 \% in Relation zum BIP zulassen. Deutschland hat sich, wie bereits erläutert, eine noch strengere Grenze von $0,35 \%$ gesetzt. Die Vertragsparteien sollen eine rasche Annäherung an ihr jeweiliges mittelfristiges Ziel sicherstellen. Daneben ist ein automatischer Korrekturmechanismus bei Zielverfehlung vorgeschrieben.

Festzuhalten ist, dass diese Reformen bereits wirken und sich zeigt, dass insbesondere die Kopplungen der Hilfszusagen an Reformbemühungen Erfolge herbeiführen. So urteilt z.B. Holger Schmieding (Chefvolkswirt der Berenberg Bank):

„Die Europäische Währungsunion bietet eine gute Stabilitätsordnung. Keinem vergleichbaren Wirtschaftsraum der westlichen Welt ist es besser gelungen, die Stabilität zu wahren und mit Reformen die Grundlage für künftiges Wachstum zu legen“. ${ }^{16}$

Nach starken aber notwendigen Anpassungsprozessen, wie z.B. einem signifikanten Rückgang der Lohnstückkosten, haben Irland, Spanien und Portugal den Rettungsschirm inzwischen wieder verlassen und stehen auf eigenen Beinen.

Die Rezession im Euroraum wird in diesem Jahr überwunden. Die Europäische Kommission rechnet in ihrer aktuellen Frühjahrsprognose für 2014 mit einem Wachstum der Wirtschaftsleistung im Euroraum um 1,2 \%. Für 2015 wird eine Festigung des wirtschaftlichen Aufschwungs mit einem BIP-Wachstum um $1,7 \%$ prognostiziert. Bei der Lage am Arbeitsmarkt ist mit einer schrittweisen Verbesserung zu rechnen. Da sich positive Wachstumsraten für gewöhnlich verzögert in einer besseren Lage am Arbeitsmarkt widerspiegeln, prognostiziert die Europäische Kommission für den Euroraum für 2015 zunächst einen leichten Rückgang der Arbeitslosenquote auf 11,4 \% gegenüber 11,8 \% in 2014. Gleichzeitig ist es gelungen, das aggregierte Haushaltsdefizit in der Eurozone deutlich zu reduzieren von 6,4\% in 2009 auf erwartete 2,5\% in 2014. Dieser Pfad muss konsequent weiter gegangen werden.

Die Erfolge des Euroraums werden offenkundig, wenn man den Blick auf andere große Industrienationen lenkt. So macht das Beispiel Japan deutlich, dass eine fortwährend expansive Geld- und Fiskalpolitik mit schuldenfinanzierten Konjunkturprogrammen kein Garant für eine dynamische wirtschaftliche Entwicklung ist. Für Japan prognostiziert die OECD im Jahr 2014 beispielsweise ein reales Wachstum des BIP von knapp 1,2\% bei einem gesamtstaatlichen Defizit 
von $8,4 \%$ in Relation zum BIP. Aktuelle Projektionen für den US-Haushalt auf Bundesebene zeigen, dass eine sinkende Schuldenstandsquote in den nächsten zehn Jahren nicht zu erwarten ist und man nicht davon ausgehen kann, dass die USA wie selbstverständlich aus ihren Schulden herauswachsen können. Für die Vereinigten Staaten schätzt die OECD für das Jahr 2014 das reale BIPWachstum auf 2,6 \% bei einem gesamtstaatlichen Defizit von 5,8 \% in Relation zum BIP. ${ }^{17}$

Doch diese ersten Erfolge in der Europäischen Union geben keinen Anlass sich auszuruhen. Eine Abkehr vom Kurs der strukturellen Konsolidierungspolitik flankiert mit Strukturreformen könnte die bisher erreichten Erfolge und eine Rückkehr des Vertrauens in die Zukunftsfähigkeit der betroffenen Volkswirtschaften zunichtemachen. Insofern sind Forderungen, angesichts einer sich abschwächenden Konjunktur die Ausrichtung der Finanzpolitik zu ändern, sowohl in Europa als auch in Deutschland nicht zielführend.

Deutschland muss weiter mit gutem Beispiel vorangehen und seine nachhaltige Konsolidierungspolitik fortsetzen. Nur so kann Deutschland auch zukünftig als Stabilitätsanker und Wachstumsmotor innerhalb der Europäischen Union wirken und dabei helfen, dass der europäische Wirtschaftsraum seine Position in der Weltwirtschaft auf Dauer behaupten kann. Die Bundesregierung wird den eingeschlagenen Kurs aktiv und engagiert weiterverfolgen.

Die öffentlichen Finanzen in Deutschland und Europa werden langfristig gestärkt aus der Krise hervorgehen. Das neue institutionelle Rahmenwerk erhöht die Stabilität und bewirkt große Anstrengungen auf der Ebene der Mitgliedstaaten. Die Regelverbindlichkeit wurde erhöht, und die Eigenanreize zu einer zukunftsgerichteten Politik wurden gestärkt.

\section{Der Bundeshaushalt in der 18. Legislaturperiode}

Solide Finanzen mit ausgeglichenen Haushalten sind für die Bundesregierung unerlässlich. Die Neuverschuldung soll dauerhaft gestoppt werden, die Schuldenstandsquote gesenkt und dabei die Investitionskraft von Bund, Ländern und Kommunen sichergestellt werden. Nur so wird die Bundesregierung heute der Verantwortung gegenüber nachfolgenden Generationen gerecht.

17 Alle Angaben gerundet auf eine Nachkommastelle. Vgl. OECD: OECD Wirtschaftsausblick, Ausgabe 2014/1, Paris, 2014. 
Die „Null“ im Haushalt 2015 ist das Ergebnis der konsequenten Haushaltskonsolidierung der letzten Jahre. In der Konsolidierungsphase hat die Bundesregierung das Ausgabenniveau konstant gehalten und die Mehreinnahmen überwiegend für den Abbau der Neuverschuldung eingesetzt.

So liegt der Bund 2015 mit dem Erreichen des Haushaltsausgleichs mit Ausgaben von 299,5 Milliarden Euro immer noch unter dem Niveau von 2010 mit 303,7 Milliarden Euro. Der 2015 erreichte Haushaltsausgleich ohne Neuverschuldung bildet zugleich die Umsetzung der vereinbarten prioritären Maßnahmen des Koalitionsvertrags ab. Dennoch bleibt der Bund in der weiteren Ausgabenentwicklung des im Juli vom Kabinett beschlossenen Finanzplans bis 2018, also über die Legislaturperiode hinaus, insgesamt deutlich unterhalb des Wachstums der Wirtschaftsleistung und setzt die Nullverschuldung fort. Damit werden die entsprechenden Vorgaben des Koalitionsvertrages erfüllt. Zugleich wird deutlich, dass sich die strikte Konsolidierung auszahlt: durch die beharrliche Sanierung des Haushalts in den vergangenen Jahren wurden die Spielräume erarbeitet, die in dieser Legislaturperiode mit den prioritären Maßnahmen des Koalitionsvertrages gestaltend genutzt werden können.

Hier zeigt sich die bereits angesprochene Dualität von Konsolidierung und wachstumsstärkender Politik. Die Konsolidierungspolitik der vergangenen Legislaturperioden ermöglicht folgende zusätzlichen Investitionen:

- Der Bund entlastet die Kommunen durch die vollständige Übernahme der Grundsicherung im Alter um weitere 1,1 Milliarden Euro und gibt den Kommunen damit mehr Spielraum für Infrastrukturinvestitionen. Diese werden schließlich zu einem großen Teil von den Kommunen vorgenommen. Die Kommunen werden außerdem im Vorgriff auf eine Reform der Eingliederungshilfe für Menschen mit Behinderung ab 2015 um 1 Milliarde Euro jährlich und nach Verabschiedung des Bundesteilhabegesetzes ab dem Jahr 2018 im Umfang von 5 Milliarden Euro pro Jahr entlastet.

- Neben der Entlastung der Gebietskörperschaften wird der Bund auch seine eigenen Investitionsmittel für die Verkehrsinfrastruktur des Bundes um 5 Milliarden Euro aufstocken und die Städtebauförderung um zusätzliche 600 Millionen Euro ausweiten.

- Ebenso werden die Gemeinden, gemeinsam mit den Ländern, in den Bereichen Bildung und Forschung entlastet. Dies soll die Kita-Ausstattung verbessern und durch das breitere Angebot von Ganztagsbetreuung die Partizipationsmöglichkeiten der Eltern am Arbeitsmarkt stärken. Des Weiteren werden die Länder in die Lage versetzt, durch die Entlastung durch den Bund in Höhe von 6 Milliarden Euro im Bildungsbereich, z.B. in Form der 
vollständigen und dauerhaften Übernahme des BAföG, ihre Aufgaben in diesem Bereich zukunftsfest zu finanzieren.

- Die gezielte Unterstützung der Forschungslandschaft zeigt sich auch in der Weiterführung des Hochschulpakts, des Pakts für Forschung und Innovation und der Exzellenzinitiative. Den Aufwuchs für die außeruniversitäre Forschung finanziert der Bund in Zukunft allein. Dazu stehen 3 Milliarden Euro zur Verfügung.

Nach der Sanierung des Haushalts geht es in den nächsten Jahren darum, das Erreichte dauerhaft zu sichern und das richtige Ausmaß von Ausgaben und Einnahmen zu wahren. Ausgehend vom Haushaltsausgleich ist es möglich, die „Null“ zu halten, die Steuereinnahmen für die richtigen Ausgabenschwerpunkte zu verwenden und dabei die Struktur des Bundeshaushalts in Richtung der auf mehr wachstumsfördernde Ausgaben zu verbessern. Damit wird zugleich deutlich, dass die Schuldenregel keine Investitionsbremse ist.

Naturgemäß beruht die Haushaltsaufstellung auf Annahmen über die zukünftige wirtschaftliche Entwicklung. Die Bundesregierung geht hier wie in den vergangenen Jahren einen vorsichtigen Weg. Die Wachstumsprojektion der Bundesregierung von real 1,8 \% für 2014 und 2,0 \% für 2015 liegt innerhalb der Prognosen der Wirtschaftsforschungsinstitute. ${ }^{18}$ Die darauf aufbauende Schätzung der Steuereinnahmen ist realistisch und sachgerecht. Sie bildet den erwartbaren Verlauf der Steuereinnahmen des Bundes nach bester Methodik ab. Dabei zeigt sich im Mehrjahresvergleich ein erfreulicher Anstieg der Steuereinnahmen des Bundes - aber auch in besonderem Maße auf Seiten der Länder und Gemeinden. Hauptgrund dafür bleibt die gute Beschäftigungslage in Deutschland. ${ }^{19}$

Mit dem am 2. Juli 2014 beschlossenen Finanzplan bis 2018 leistet der Bund einen entscheidenden Beitrag zur dauerhaften Einhaltung des europäischen Regelwerks und damit zur Stabilität und zur Sicherung der Wachstumschancen in Europa. Der gesamtstaatliche Finanzierungssaldo in der Maastricht-Rechnung, der neben dem Bund auch die Länder, Gemeinden und Sozialversicherungen umfasst, ist bereits seit 2012 im Überschuss. Dies wird nach allen Prognosen auch in den nächsten Jahren so bleiben. Die Zeit, als Deutschland sich mit Defiziten von weit über der Obergrenze von $3 \%$ des BIP einem Defizitverfahren der Europäischen Kommission stellen musste, ist überwunden. Heute ist Deutsch-

18 Vgl. Projektgruppe Gemeinschaftsdiagnose: Gemeinschaftsdiagnose Frühjahr 2014, 2014.

19 Eine detaillierte Darstellung zur jüngsten Steuerschätzung findet sich in Bundesministerium der Finanzen: Ergebnisse der Steuerschätzung vom 6. bis 8. Mai 2014, in: BMF-Monatsbericht Mai 2014 (2014), abrufbar unter: http://www.bundesfinanzministerium.de. 
land das einzige Land im Euroraum, das einen positiven Finanzierungssaldo aufweist.

$\mathrm{Zu}$ diesem Ergebnis trugen nicht zuletzt die Finanzierungsüberschüsse der Sozialversicherungen und der kommunalen Ebene in den zurückliegenden Jahren bei, die vom Bund massiv finanziell unterstützt wurden. Gerade an den Kommunen zeigt sich, dass Zukunftsvorsorge einerseits den Verzicht auf neue Schulden und andererseits kluges Investieren bedeutet. Nicht zuletzt aufgrund der finanziellen Entlastungen durch den Bund, etwa bei der vollständigen Übernahme der Finanzierungslasten für die Grundsicherung im Alter, haben die Gemeinden in Deutschland ihre Investitionen im vergangenen Jahr in der Abgrenzung der volkswirtschaftlichen Gesamtrechnung um rund $9 \%$ gegenüber 2012 ausweiten können und gleichzeitig ihre - bundesweit betrachtet - sehr gute Haushaltslage bewahrt.

\section{Sicherung der Sozialsysteme}

Der Bundeshaushalt bleibt auch in den nächsten Jahren ein Garant für die Funktionsfähigkeit der sozialen Sicherungssysteme in Deutschland. Jeder zweite Euro geht an die Sozialversicherungen. Die Mittel für die gesetzliche Rentenversicherung und die gesetzliche Krankenversicherung werden von 93,5 Milliarden Euro im laufenden Jahr auf rund 108 Milliarden Euro im Jahr 2018 anwachsen. Den Gesundheitsfonds hat der Bund seit seiner Errichtung zum 1. Januar 2009 mit insgesamt mehr als 70 Milliarden Euro unterstützt. Auch dies hat dazu beigetragen, dass das System der gesetzlichen Krankenversicherung inzwischen Reserven von mehr als 30 Milliarden Euro bilden konnte. Der Bund bleibt ein verlässlicher Partner für die gesetzlichen Krankenversicherungen. So steigt der Bundeszuschuss an den Gesundheitsfonds von 10,5 Milliarden Euro im Jahr 2014 über 11,5 Milliarden Euro im Jahr 2015 auf 14 Milliarden Euro im Jahr 2016 und 14,5 Milliarden Euro ab 2017. Die leichte Absenkung des Zuschusses gegenüber den bisherigen Planungen in den Jahren 2014 und 2015 ist angesichts der sehr positiven Finanzlage im Gesundheitsfonds sinnvoll. Es wäre schwer vermittelbar und zudem auch unwirtschaftlich, hohe Überschüsse im Gesundheitssystem durch Aufnahme neuer Schulden im Bundeshaushalt zu finanzieren.

\section{Bund-Länder-Finanzbeziehungen nach 2019}

Die föderale Gliederung der Bundesrepublik Deutschland macht es unumgänglich, die Finanzpolitik des Bundes innerhalb dieser Strukturen und nicht singulär zu betrachten und zu diskutieren. Vor diesem Hintergrund - und insbesondere 
wegen des Auslaufens bedeutender Regelung für die finanziellen Beziehungen zwischen den Gebietskörperschaften - wäre diese Abhandlung unvollständig ohne einen Ausblick auf die anstehenden Verhandlungen zur Neuordnung der föderalen Finanzbeziehungen. Dies gilt umso mehr, als die oben beschriebenen Reformen der fiskalischen Regeln (grundgesetzliche Schuldenregel sowie Stabilitäts- und Wachstumspakt) eine gesamtstaatliche Perspektive haben und ihre Erfüllung stets die gemeinsame Aufgabe aller Gebietskörperschaften ist.

Die föderalen Finanzbeziehungen waren stets Ausdruck praktizierter Solidarität in Deutschland: Deshalb ist es gelungen, die Herausforderungen vieler Umbrüche erfolgreich zu gestalten, insbesondere die Begleitung der deutschen Einheit. Um auch zukünftig die Handlungsfähigkeit sicherzustellen, muss das System jedoch von Zeit zu Zeit auf den Prüfstand gestellt werden. Der Koalitionsvertrag enthält diesbezüglich einen klaren Auftrag. Hier wurde verankert, dass unser föderales System als Grundlage der Demokratie und Leistungsfähigkeit Deutschlands weiterhin handlungsfähig bleiben muss. Jede Ebene - Bund, Länder und Kommunen - soll ihren Aufgaben mit einem hohen Maß an Eigenverantwortung nachkommen können. Damit wird einer Neuordnung der Bund-LänderFinanzbeziehungen in dieser Legislaturperiode eine hohe Bedeutung zukommen. Dabei muss das gemeinsame Ziel handlungsleitend sein, dass der Bund und alle Länder die grundgesetzliche Schuldenregel einhalten. Dies bedeutet noch erhebliche Anstrengungen, trotz der auf diesem Weg bereits erzielten Erfolge. Der Bund hat seinen Beitrag stets geleistet, und er wird auch in Zukunft als verlässlicher Partner seiner Verantwortung im föderalen System gerecht werden.

Die derzeitigen Regelungen für das Ausgleichssystem wurden im Jahr 2001 einvernehmlich vom Bund und allen Ländern vereinbart und sind seit 2005 praktisch unverändert. Weil die Höhe der Ausgleichsströme vor allem von der Entwicklung der Steuereinnahmen in den einzelnen Ländern bestimmt wird, ist das Volumen der Umverteilung in erster Linie Spiegelbild der Steuerkraftunterschiede zwischen den Ländern. Die Steuerkraft in den ostdeutschen Ländern (ohne Umsatzsteuer) und ihren Gemeinden liegt nach wie vor bei weniger als zwei Dritteln des Bundesdurchschnitts bzw. bei lediglich knapp der Hälfte des Niveaus der aktuellen Zahlerländer im Länderfinanzausgleich. Folglich geht auch der weit überwiegende Teil der Ausgleichsleistungen in Richtung ostdeutscher Länder.

Die föderale Einnahmeverteilung in Deutschland ist Ergebnis eines mehrstufigen Verteilungs- und Umverteilungsprozesses. Mit der vertikalen Steuerverteilung wird die Aufteilung der Steuereinnahmen auf den Bund und die Ländergesamt- 
heit sowie die Gemeindeebene festgelegt. Die horizontale Verteilung der auf die Ländergesamtheit entfallenden Steuereinnahmen auf die einzelnen Länder erfolgt grundsätzlich nach dem örtlichen Aufkommen dieser Einnahmen; sie stehen also den einzelnen Ländern zu, die sie vereinnahmen. Einige Steuern werden dabei über so genannte Zerlegungen nachträglich richtig zugeordnet.

Auf dieser Grundlage beruht das Ausgleichssystem, bestehend aus der horizontalen Verteilung des Länderanteils an der Umsatzsteuer über Ergänzungsanteile, dem horizontalen Finanzausgleich zwischen den Ländern auf Grundlage der Finanzkraft der Länder (Länderfinanzausgleich i.e.S.) sowie den allgemeinen Bundesergänzungszuweisungen und Sonderbedarfsbundesergänzungszuweisungen. Aufgabe des horizontalen Finanzausgleichsystems ist es, die insgesamt zur Verfügung stehenden Finanzmittel so auf die einzelnen Länder aufzuteilen, dass alle in die Lage versetzt werden, die ihnen verfassungsrechtlich vorgegebenen Aufgaben zu erfüllen.

Der Finanzausgleich ist dabei auf den Durchschnitt der Finanzkraft der Länder ausgerichtet: Länder mit überdurchschnittlicher Finanzkraft sind ausgleichspflichtig, Länder mit unterdurchschnittlicher Finanzkraft ausgleichsberechtigt. Die Ausgleichsleistungen sind damit primär Ersatz für fehlende eigene Steuereinnahmen empfangsberechtigter Länder. Darüber hinaus dient ein Teil der Leistungen des Ausgleichsystems dem Ausgleich von Sonderlasten einzelner Länder.

Ende 2019 laufen die einschlägigen Gesetze zum Bund-Länder-Finanzausgleich aus. Oberstes Ziel einer Neuordnung der föderalen Finanzbeziehungen sollte das Erreichen einer Balance sein - einer Balance zwischen der im Grundgesetz verankerten Solidarität im Bundesstaat, die in der Vergangenheit auch durch Bundesverfassungsgerichtsurteile bestätigt wurde, der Effizienz, Transparenz und Gerechtigkeit des Systems sowie der Eigenverantwortung der Länder. Deshalb ist bei der Neuordnung auch die Frage zu diskutieren, wie die ökonomischen Anreize des Systems verbessert werden können, ohne das verfassungsrechtliche Ziel der Gleichwertigkeit der Lebensverhältnisse in Frage zu stellen. Denn wie im Koalitionsvertrag festgelegt, sollte eine Neuordnung die Voraussetzungen schaffen, dass eine nachhaltige Konsolidierung der Länderhaushalte erfolgt und die ab 2020 für alle Länder verbindlich geltende grundgesetzliche Schuldenregel dauerhaft aus eigener Kraft eingehalten werden kann.

Für eine Einigung zu Lasten des Bundes sieht die Bundesregierung keinen Spielraum. Ungeachtet der Vorgabe des Grundgesetzes, nach der Bund und Länder gleichmäßigen Anspruch auf Deckung ihrer notwendigen Ausgaben durch lau- 
fende Einnahmen haben, zeigt die Finanzentwicklung von Bund und Ländern seit langem eine deutliche Schieflage zu Lasten des Bundes. Diese hat sich als Folge der globalen Finanz- und Wirtschaftskrise in den letzten Jahren sogar noch verstärkt und kumuliert in einem Schuldenstand des Bundes, der zum Jahresende 2013 mit 1.090 Milliarden Euro rund das Doppelte der Schuldenlast der Länder betrug.

Pro Kopf liegen die Schulden des Bundes in etwa auf dem saarländischen Niveau. Lediglich Berlin und Bremen weisen schlechtere Zahlen auf. Dementsprechend stark ist der Bundeshaushalt mit Zinszahlungen belastet. 2013 zahlte der Bund 31,3 Milliarden Euro Zinsen. Das entspricht 10,2 \% des Bundeshaushalts. Im Durchschnitt der Landeshaushalte sind dagegen nur 5,7\% der Ausgaben Zinslasten. Dem Ziel nominal ausgeglichener Haushalte sind die Länder mit einem Finanzierungssaldo ihrer Kernhaushalte von insgesamt 2,3 Milliarden Euro deutlich näher als der Bund, der im vergangenen Jahr noch ein Defizit von 22,3 Milliarden Euro auswies.

Trotz dieser Schieflage hat der Bund die Länder und Kommunen auch in der 17. Legislaturperiode bei der Wahrnehmung ihrer Aufgaben unterstützt. Für die 18. Legislaturperiode sieht der Koalitionsvertrag, wie bereits erläutert, ${ }^{20}$ weitere finanzielle Entlastungen der Länder und Kommunen durch den Bund in einer Größenordnung von über 10 Milliarden Euro als prioritäre Maßnahmen vor. ${ }^{21}$ Ein solcher Betrag sollte klar machen, dass sich der Bund seiner Verantwortung gegenüber den Ländern und Kommunen stellt und seinen fairen Finanzierungsanteil übernimmt.

Um nach einer Neuordnung der föderalen Finanzbeziehungen ein langfristig tragfähiges System zu erhalten, ist eine Prüfung der Aufgaben-, Ausgaben- und Einnahmenverteilung zwischen den föderalen Ebenen angebracht. Eine geeignete Zuordnung von Aufgaben und Einnahmequellen kann eine eigenverantwortliche Aufgabenwahrnehmung unterstützen. Eine Aufgabe sollte dabei von der staatlichen Ebene erfüllt und finanziert werden, die diese am besten und effizientesten bereitstellen kann. Müssen also z.B. unterschiedliche Präferenzen vor Ort in Betracht gezogen werden, sollte die Aufgabe auf Länder- bzw. Gemeindeebene übernommen werden. Ist hingegen eine einheitliche Erfüllung von gesamtstaatlich bedeutsamen Aufgaben angezeigt, ist der Bund gefragt. Dies entspräche

20 Siehe hierzu die Bullet-Points in Abschnitt IV.

21 Wobei Regelungen wie die im zu verabschiedenden Bundesteilhabegesetz über die Legislatur hinaus wirksam sind. 
auch der Vorgabe des Grundgesetzes, das eine Wahrung der Gleichwertigkeit der Lebensverhältnisse im Bundesgebiet vorgibt. Reformen bei der Aufgabenverteilung sollten dabei durch Veränderungen bei der Einnahmenverteilung begleitet werden. So könnten die Unterschiede bei der Finanzkraft der Länder besser angeglichen und den Ländern auch hier mehr Autonomie gewährt werden.

Die Formulierung von Maximalforderungen darf nicht zum Scheitern der Verhandlungen führen. Es gilt deshalb die divergierenden Interessen zu überbrücken. Die Bundesregierung ist zuversichtlich, dass der Bund gemeinsam mit den Ländern in dieser Legislaturperiode die Weichen für eine nachhaltige Neuordnung der Bund-Länder-Finanzbeziehungen zur Zufriedenheit aller Beteiligten stellen kann. Dies wäre ein bedeutendes Vertrauenssignal an unsere Bürgerinnen und Bürger für eine zukunftsfähige föderale Ordnung. Zugleich wäre dies ein wichtiger Schritt für ein nachhaltiges Einhalten der grundgesetzlichen Schuldenregel sowohl auf Bundes- als auch auf Landesebene. Ein Bekenntnis aller föderalen Ebenen zu dauerhafter finanzpolitischer Solidität hat nicht nur national große Bedeutung, sondern ist auch wichtig für unsere Vorbild- und Ankerfunktion in Europa.

\section{Fazit}

Die Einführung der grundgesetzlichen Schuldenregel und die Stärkung des Stabilitäts- und Wachstumspakts zeigen Wirkung. Die öffentlichen Finanzen in Deutschland und Europa werden langfristig gestärkt aus der Krise hervorgehen. Das neue institutionelle Rahmenwerk erhöht die Stabilität und bewirkt große Anstrengungen auf der Ebene der Mitgliedstaaten. Die Regelverbindlichkeit wurde erhöht und die Eigenanreize zu einer zukunftsgerichteten Politik wurden gestärkt.

Die Konsolidierung zahlt sich aus. Deutschland ist dazu in der Lage, seine gewonnenen Spielräume für prioritäre Maßnahmen zu nutzen und so die Finanzpolitik wachstumsorientierter auszugestalten. Die Ausweitungen der Investitionen finden in allen Gebietskörperschaften statt und legen so die Basis für ein langfristiges Wachstum und eine leistungsfähige Infrastruktur.

Die Entlastung der Länder- und Gemeindehaushalte versetzt diese in die Lage, auf einem soliden Fundament die anstehende Neuordnung der föderalen Finanzbeziehungen fair und transparent für die Zeit nach 2019 zu gestalten. 